\title{
Tuning thermoelectric efficiency of monolayer indium nitride by mechanical strain
}

Cite as: J. Appl. Phys. 129, 234302 (2021); https://doi.org/10.1063/5.0051461

Submitted: 25 March 2021 • Accepted: 28 May 2021 • Published Online: 15 June 2021

(iD) M. M. Cicek, M. Demirtas and (iD) E. Durgun
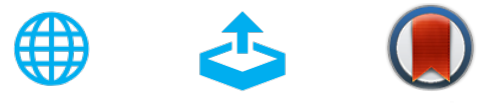

View Online

Export Citation

\section{ARTICLES YOU MAY BE INTERESTED IN}

Forward and reverse current transport mechanisms in tungsten carbide Schottky contacts on AlGaN/GaN heterostructures

Journal of Applied Physics 129, 234501 (2021); https://doi.org/10.1063/5.0052079

Interfacial engineering for the enhancement of interfacial thermal conductance in GaN/AIN heterostructure

Journal of Applied Physics 129, 235102 (2021); https://doi.org/10.1063/5.0052742

Optical cloaking and invisibility: From fiction toward a technological reality

Journal of Applied Physics 129, 231101 (2021); https://doi.org/10.1063/5.0048846

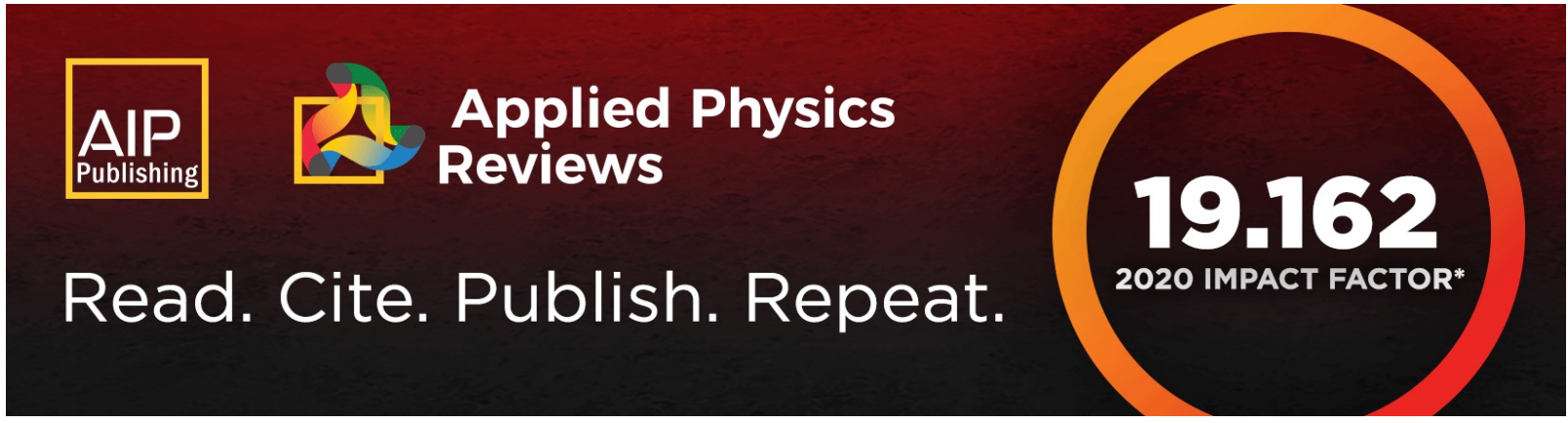




\title{
Tuning thermoelectric efficiency of monolayer indium nitride by mechanical strain
}

\author{
Cite as: J. Appl. Phys. 129, 234302 (2021); doi: $10.1063 / 5.0051461$ \\ Submitted: 25 March 2021 . Accepted: 28 May 2021 . \\ Published Online: 15 June 2021
}

M. M. Cicek, ${ }^{1,2}$ (D) M. Demirtas, ${ }^{7}$ and E. Durgun ${ }^{1, a)}$ (D)

\begin{abstract}
AFFILIATIONS
${ }^{1}$ UNAM-National Nanotechnology Research Center and Institute of Materials Science and Nanotechnology, Bilkent University, Ankara 06800, Turkey

${ }^{2}$ Department of Engineering Physics, Faculty of Engineering, Ankara University, Ankara 06100, Turkey
\end{abstract}

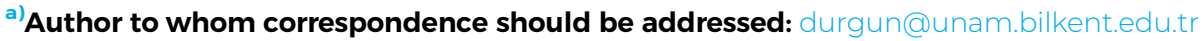

\begin{abstract}
Tuning the thermoelectric efficiency of a material is a complicated task as it requires the control of interrelated parameters. In this respect, various methods have been suggested to enhance the figure of merit (ZT), including the utilization of low-dimensional systems. Motivated by the effect of strain on intrinsic properties of two-dimensional materials, we examine the thermoelectric response of monolayer indium nitride $(\mathrm{h}-\mathrm{InN})$ under low biaxial strain $( \pm 1 \%)$ by using ab initio methods together with solving Boltzmann transport equations for electrons and phonons. Our results indicate that among the critical parameters, while the Seebeck coefficient is not affected prominently, electrical conductivity can increase up to three times, and lattice thermal conductivity can decrease to half at $-1 \%$ strain where valence band convergence is achieved. This results in significant enhancement of ZT, especially for p-type h-InN, and it reaches 0.50 with achievable carrier concentrations $\left(\sim 10^{13} \mathrm{~cm}^{-2}\right)$ at room temperature. Thermoelectric efficiency further increases with elevated temperatures and rises up to 1.32 at $700 \mathrm{~K}$, where the system remains to be dynamically stable, suggesting h-InN as a promising material for high-temperature thermoelectric applications.
\end{abstract}

Published under an exclusive license by AIP Publishing. https://doi.org/10.1063/5.0051461

\section{INTRODUCTION}

Thermoelectric energy conversion that can convert waste heat directly into electricity (and vice versa) has drawn attention over the past few decades as a promising energy harvesting strategy. ${ }^{1,2}$ The efficiency of thermoelectric materials is evaluated by the dimensionless figure of merit, $\mathrm{ZT}=S^{2} \sigma T / \kappa$, where $S$ is the Seebeck coefficient, $\sigma$ is the electrical conductivity, $S^{2} \sigma$ is the power factor (PF), $T$ is the absolute temperature, and $\kappa$ is the sum of electronic $\left(\kappa_{e}\right)$ and lattice $\left(\kappa_{l}\right)$ thermal conductivity. ${ }^{3}$ Thermoelectric materials with high efficiency should simultaneously have a large PF and a low $\kappa$, which is a challenging task due to the interdependence of these parameters. Therefore, several approaches have been suggested to enhance ZT, including nanostructuring, ${ }^{4,5}$ band engineering, ${ }^{3,6-8}$ and chemical modification. ${ }^{9,10}$ However, the desired efficiency for high technological devices to be integrated into a vast range of sectors could not have been achieved yet. ${ }^{1}$

An alternative and promising strategy to optimize ZT is utilizing two-dimensional (2D) materials as reduced dimensions, which can lead to better thermoelectric conversion efficiency compared to the bulk counterparts. ${ }^{12-14}$ For instance, a decrease in the thickness (i.e., confinement length) and the thermal de Broglie wavelength can enhance the PF. ${ }^{13}$ Together with this, electrons and phonons have different mean free paths due to the confinement, which allows engineering their contributions to thermal conductivity individually. ${ }^{12}$ Accordingly, superior thermoelectric properties of several 2D materials have been demonstrated ${ }^{14-16}$ and their potential to be used in thermoelectric devices is revealed. ${ }^{17,18}$ Moreover, 2D semiconductors naturally offer high tunability of thermoelectric response with chemical modification, strain, and stacking. ${ }^{18-24}$

Within the quest for identifying potential 2D materials for thermoelectric applications, the class of group III-nitrides that possess novel electronic and optical properties ${ }^{25}$ are also taken into consideration. Although monolayers of $\mathrm{BN}, \mathrm{AlN}$, and $\mathrm{GaN}$ have been reported to have low ZT values, the relatively high (low) electrical (thermal) conductivity of monolayer hexagonal indium nitride (h-InN) leads to good thermoelectric efficiency. ${ }^{26,27}$ Albeit 2D InN has not been realized yet, the stability of h-InN has been revealed and its fundamental properties have been characterized. ${ }^{28,29}$ 
Additionally, the synthesis of InN in thin film and nanostructure forms has been achieved and their thermoelectric properties have been investigated. ${ }^{30-33}$ Accordingly, h-InN holds the promise to be used in thermoelectric applications subsequent to further enhancement of its intrinsic thermoelectric efficiency.

With this motivation, in this work, we study the effect of low mechanical strain on the thermoelectric response of h-InN by using first-principle methods and Boltzmann transport theory. First, the optimized geometries and corresponding electronic band structures are obtained under compressive/tensile strain $( \pm 1 \%)$. Next, the strain-dependent relaxation time $(\tau)$, which is required to include the effect of scattering mechanisms (i.e., electron-phonon coupling), is estimated. The variation of these coefficients [Seebeck coefficient $(S)$, electrical conductivity $(\sigma)$, power factor $\left(S^{2} \sigma\right)$, and electronic thermal conductivity $\left.\left(\kappa_{e}\right)\right]$ with carrier concentration (electron and hole) are calculated for each strain value. To calculate the phonon transport properties, a primarily accurate phonon spectrum is obtained and then the alteration of lattice thermal conductivity $\left(\kappa_{l}\right)$ with strain and temperature is examined. Finally, the figure of merit $(\mathrm{ZT})$ under strain is calculated for varying temperature and carrier concentration, and significant enhancement in thermoelectric efficiency of p-type h-InN under low compressive strain is revealed.

\section{METHOD}

The first principles calculations based on density functional theory (DFT) were performed using Vienna ab initio simulation package (VASP). ${ }^{34-37}$ Perdew-Burke-Ernzerhof (PBE) representation of the generalized gradient approximation (GGA) was employed to describe the exchange-correlation functional. ${ }^{38}$ The electronic band structures were also calculated with the inclusion of spin-orbit coupling (SOC). ${ }^{39}$ The projector augmented wave $(\mathrm{PAW})^{40,41}$ potentials (including semi-core $d$-electrons) with a kinetic energy cutoff of $550 \mathrm{eV}$ was used. The Brillouin zone (BZ) was sampled with $15 \times 15 \times 1 \mathrm{k}$-point mesh during the structural relaxation until the energy difference between two electronic steps was smaller than $10^{-6} \mathrm{eV}$ and the maximum Hellmann-Feynman force acting on each atom was less than $10^{-5} \mathrm{eV} / \AA$. The vacuum space of $20 \AA$ was set to eliminate the long-range interactions between the periodic images. The crystal structures were visualized by VESTA software. ${ }^{42}$

The electronic parts of the thermoelectric transport coefficients were calculated by solving the semiclassical Boltzmann transport equation (BTE) by using the BoltzTraP code. ${ }^{43,44}$ In the frame of the Boltzmann transport theory, the electronic transport coefficients can be revealed as

$$
\begin{gathered}
S_{\alpha \beta}(T, \mu)=\frac{1}{e T V \sigma_{\alpha \beta}(T, \mu)} \int \sigma_{\alpha \beta}(E)(E-\mu)\left[-\frac{\partial f_{\mu}(T, E)}{\partial E}\right] d E, \\
\sigma_{\alpha \beta}(T, \mu)=\frac{1}{V} \int \sigma_{\alpha \beta}(E)\left[-\frac{\partial f_{\mu}(T, E)}{\partial E}\right] d E
\end{gathered}
$$

where $\alpha$ and $\beta$ are the tensorial indices; $E, T, V$, and $\mu$ are the electron band energy, temperature, volume of the unit cell, and chemical potential, respectively; $f_{\mu}$ is the Fermi distribution function.
The $\sigma_{\alpha \beta}(E)$ is the energy projected conductivity tensors and can be calculated from the accurate band structure formula

$$
\sigma_{\alpha \beta}(E)=\frac{1}{N} \sum_{i, k} \tau(i, k) v_{\alpha}(i, k) v_{\beta}(i, k) \delta[E-E(i, k)],
$$

where $N$ the number of k-points used in the calculation, $i$ is the band index, $\tau(i, k)$ is the relaxation time, $v_{\alpha}(i, k)$ is the $\alpha$ component of the band velocity, and $E(i, k)$ is the energy of the state at $\mathrm{k}$-point in the $i$ th band.

A denser k-point mesh $(60 \times 60 \times 1)$ was used to obtain accurate Fourier interpolation of the Kohn-Sham eigenvalues, which is used for solving BTE under the constant relaxation time approximation in terms of carrier concentration. The spin-orbit coupling (SOC) was not included in the calculations of transport properties, as the band dispersion energies near the Fermi level were not altered dramatically when SOC was taken into account (Fig. S1 in the supplementary material). The electronic transport coefficients $S, \sigma / \tau$, and $K_{e} / \tau$ were calculated as a function of carrier concentration and temperature for each biaxial strain. To obtain $\mathrm{ZT}^{45}$ the relaxation time $(\tau)$ was calculated by using deformation potential theory. ${ }^{46}$ The theory relies on the coupling between electrons and acoustic phonons (one of the dominant scattering mechanism ${ }^{47}$ ) and is widely used in semiconductors, ${ }^{48,49}$

$$
\tau=\left(\mu \cdot m^{*}\right) / e,
$$

with the band effective mass $\left(m^{*}\right)$ defined as ${ }^{50}$

$$
m^{*}=\frac{\hbar^{2}}{d^{2} E(k) / d k^{2}}
$$

and the carrier mobility $(\mu)$ estimated as

$$
\mu=\frac{2 e \hbar^{3} Y_{2 D}}{3 k_{B} T m^{*} E_{d}^{2}},
$$

where $\hbar$ is the reduced Planck constant, $k$ is the wave vector, $E(k)$ is the energy of an electron at wavevector $k$ in the corresponding band, $e_{2}$ is the electron charge, $Y_{2 D}$ is the in-plane stiffness $\left(Y_{2 D}=\frac{c_{11}^{2}-c_{12}^{2}}{c_{11}}\right.$, where $c_{i j}$ 's are the elastic constants), $k_{B}$ is the Boltzmann constant, $T$ is the temperature, and $E_{d}$ is the deformation potential constant. The calculated $Y_{2 D}$ for h-InN is $65 \mathrm{~N} / \mathrm{m}$ and $E_{d}$ 's for hole and electrons are 5 and $20 \mathrm{eV}$, respectively.

The lattice thermal conductivity $\left(\kappa_{l}\right)$ was obtained iteratively followed by calculation of the harmonic and anharmonic force constants based on the zeroth and fully iterative solution of the Boltzmann transport equation considering phonon-phonon interaction $^{51}$ implemented in ShengBTE packages, ${ }^{52}$ which takes dominant phonon scattering mechanisms into account (i.e., three-phonon processes and isotopic disorder). The strain-dependent phonon spectra were calculated by using the finite displacement method within $5 \times 5 \times 1$ supercells. ${ }^{53}$ Taking fifth nearest-neighbor interactions into account was tested to be sufficient to obtain converged anharmonic force constants. The $50 \times 50 \times 1$ q-point grid along with the largest van der Walls thickness (3.74 $\AA$ ) was used. The Born effective 
charges and dielectric constants were derived based on the density functional perturbation theory (DFPT), which was added as a correction to the dynamic matrix to take long-range electrostatic interactions into consideration.

\section{RESULTS AND DISCUSSION}

\section{A. Crystal structure and electronic properties}

The optimized crystal structure of h-InN in the absence of strain is shown in Fig. 1(a). Similar to the other group III-N monolayers, h-InN has a planar honeycomb structure and belongs to the P-6M2 space group. ${ }^{25}$ The bond distance $\left(\mathrm{d}_{\text {In-N }}\right)$ and the lattice constant $(|\vec{a}|)$ are calculated as 2.07 and $3.59 \AA$, respectively. The total charge density isosurface [Fig. 1(b)] represents the distribution of the electrons over the hexagonal lattice and reveals the strong in-plane $\sigma$-bonds upon hybridization of $s p^{2}$ orbitals. The planarity is sustained by $\pi$-bonds formed by $p_{z}$ orbitals perpendicular to the lattice plane. ${ }^{25}$ Bader analysis indicates a charge transfer of $1.39|e|$ from In to $\mathrm{N}$, which is expected due to electronegativity difference between cation and anion atoms. The electronegativity difference also leads to polarization of In-N bonds as revealed by the electron localization function (ELF) shown in Fig. 1(c).

The electronic band structure of h-InN along the symmetry directions of the hexagonal $\mathrm{BZ}$, as well as the corresponding orbital projected density of states (PDOS), is shown in Fig. 2 (the electronic band structures calculated with the inclusion of SOC are shown in Fig. S1 in the supplementary material). The valence band maximum and the conduction band minimum occur at the $\mathrm{K}$ and $\Gamma$ symmetry points, respectively, and the indirect bandgap $\left(E_{g-i}\right)$ is calculated as $0.56 \mathrm{eV}(1.60 \mathrm{eV})$ within the PBE (HSE06) level. The highest valence band (HVB) is relatively flat, resulting in a high density of states (DOS) near the Fermi level $\left(E_{F}\right)$, and originates mainly from the N-p orbital. The lowest conduction band (LCB) is primarily composed of In- $p$ and $\mathrm{N}-s$ orbital and more dispersive.

To reveal the thermoelectric response of strained h-InN, we begin with the variation of electronic band structure with biaxial strain within $\pm 1 \%$. For $2 \mathrm{D}$ systems, practically biaxial strain can be a)
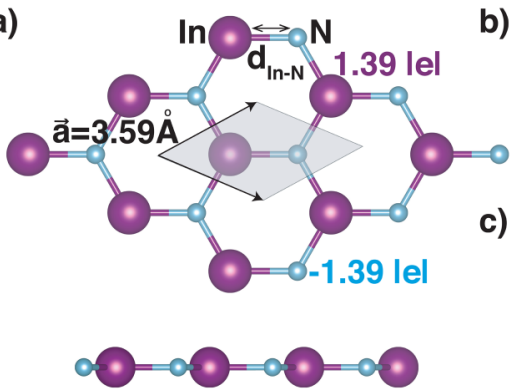

b)

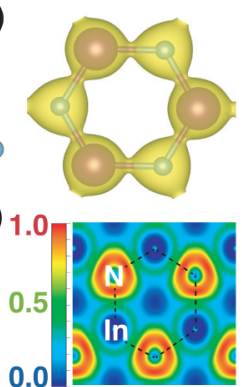

FIG. 1. (a) The top and side views of the crystal structure of $h$-InN. The purple and light blue spheres indicate In and $\mathrm{N}$ atoms, respectively. The net charge on each atom is given and the primitive unit cell is represented by the solid arrows. (b) The isosurface of the total charge density (isosurface level is set to $0.03 \mathrm{e}^{-3}$ ) and (c) in-plane electron localization function (ELF). The increase in electron localization from 0 to 1 is indicated with blue to red color code.

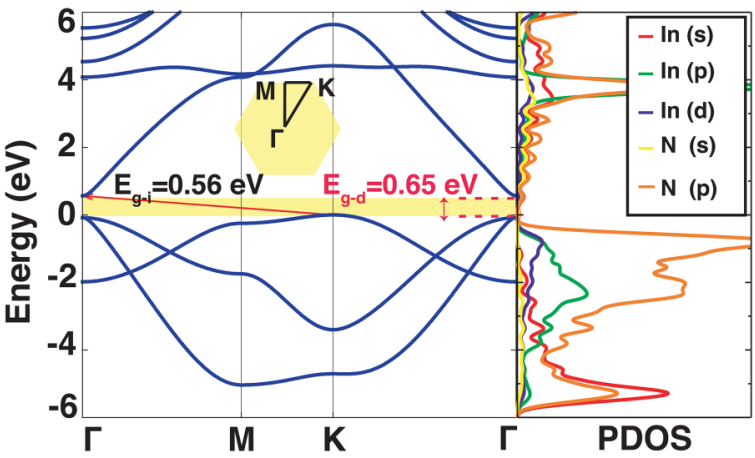

FIG. 2. Electronic band structure and orbital projected density of states (PDOS) of monolayer $\mathrm{h}$-InN. The fundamental bandgap is shaded and the indirect $\left(E_{g-i}\right)$ and direct bandgap $\left(E_{g-d}\right)$ are shown.

more easily applied by considering the lattice mismatch between 2D materials and substrates. ${ }^{54}$ The electronic structure calculations are kept at the PBE level as the band dispersion captures the transport properties and it is more crucial than the bandgap. Although the PBE underestimates the bandgap, it is reliable to calculate the thermoelectric coefficients since the band profile can be obtained with sufficient accuracy. It should be noted that apart from the bandgap, the electronic band structures calculated with PBE and HSE06 have the same profile. In this respect, the shifts of the LCB and HVB under biaxial strain are shown in Fig. 3. These shifts lead to a monotonic increase (decrease) of indirect $\left(E_{g-i}\right)$ and direct bandgap $\left(E_{g-d}\right)$ with compressive (tensile) strain. Furthermore, the difference between them narrows with compressive strain and merges at $-1 \%$. ${ }^{55}$ The LCB is composed of a single parabolic valley and undergoes notable shifts in energy as well as becomes less dispersive as applied strain varies from $+1 \%$ to $-1 \%$. On the other

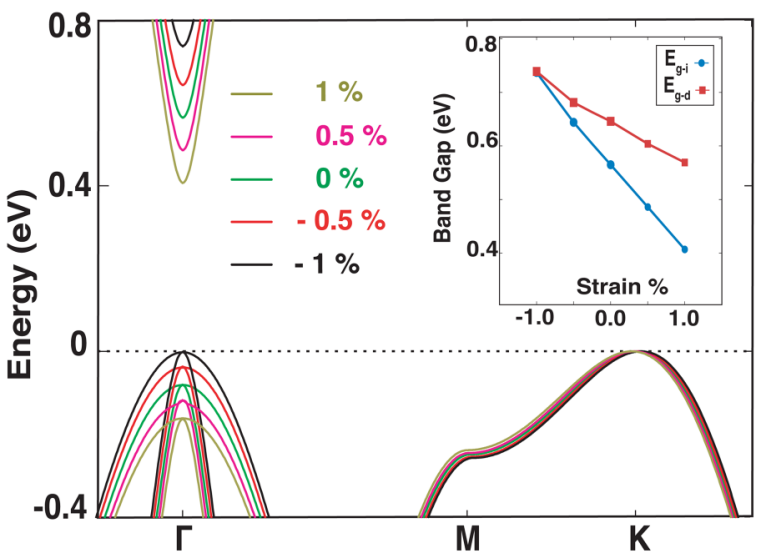

FIG. 3. The variation of the valence and conduction bands in the proximity of the Fermi level $\left(E_{F}\right)$ with biaxial strain. The inset illustrates the change of the indirect $\left(E_{g-i}\right)$ and direct bandgap $\left(E_{g-d}\right)$ under the biaxial strain. 
hand, the valence band profile in the proximity of $E_{F}$ is more complex. There are twofold degenerate bands at the $\Gamma$-point, which are located at a slightly lower energy level than the HVB at the K-point. With compressive strain, while HVB at the K-point remains almost intact, the bands at the $\Gamma$ point move upward and reduce the energy difference with HVB. This leads to a band convergence between $-0.5 \%$ and $-1 \%$ resulting in an increasing band degeneracy (i.e., the band extrema of the multiple bands have the same or comparable energy within a few $\left.k_{B} T\right){ }^{7}$ In addition to band convergence, these bands are flattened, which also affects the electronic transport features (see below). When compressive strain increases beyond $-1 \%$, energy bands at $\Gamma$-point replace the original HVB and band degeneracy gradually disappears. Additionally, we also calculated the electronic band structure of h-InN under uniaxial strain $( \pm 1 \%)$. The obtained results are reported in Fig. S5 in the supplementary material. It is noticed that the profile of band structures (or evaluation of band dispersions) is similar under both types of strain. On the other hand, the band convergence under compressive strain is less evident for the uniaxial case.

From the strain-dependent electronic band structures, the band effective mass $\left(m^{*}\right)^{50}$ and carrier mobility $(\mu)$ of charge carriers are obtained [Eqs. (5) and (6)] and these quantities can be used to estimate the relaxation time $(\tau)$ by using Eq. (4). The $m^{*}$ and $\mu$ are calculated separately for hole $\left(m_{h}^{*}, \mu_{h}\right)$ and electron $\left(m_{e}^{*}, \mu_{e}\right)$ carriers at $300 \mathrm{~K}$, and their variation with the biaxial strain is given in
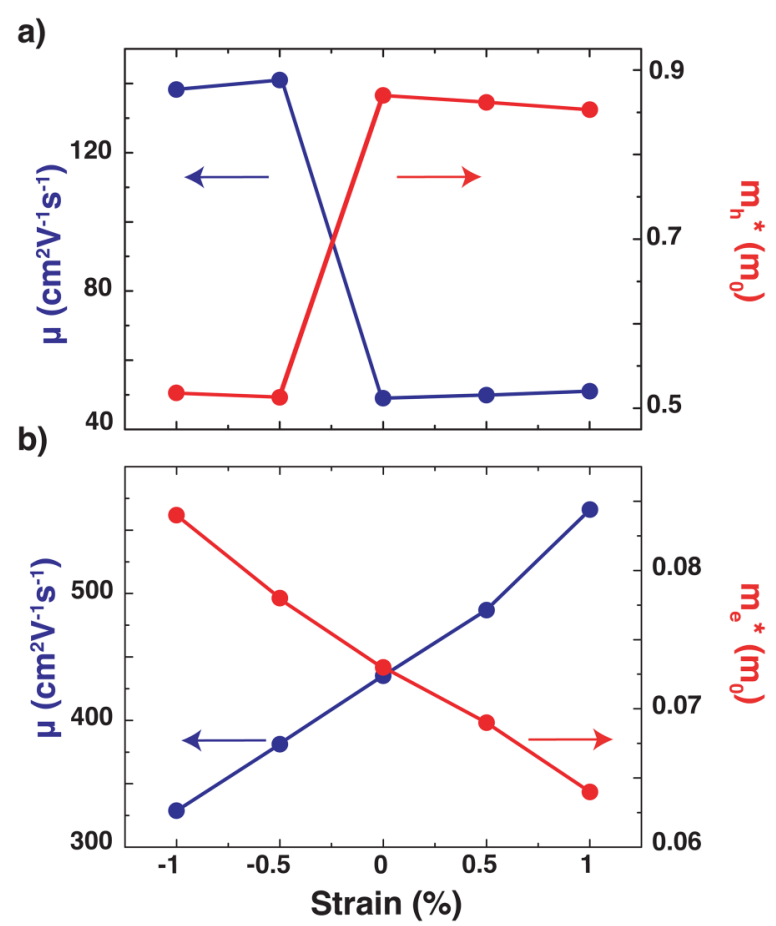

FIG. 4. The band effective mass $\left(m^{*}\right)$ and carrier mobility $(\mu)$ of $h-I n N$ at $300 \mathrm{~K}$ for (a) p-type (hole carriers) and (b) n-type doping (electron carriers) as a function of the applied biaxial strain.
Figs. 4(a) and 4(b). For all strain levels, $m_{h}^{*}$ is higher than $m_{e}^{*}$, which can be attributed to more dispersive LCB with respect to HVB. In a similar manner, as $\mu \propto 1 / m^{*}, \mu_{h}$ is lower than $\mu_{e}$ within these strain levels. The sharp decrease (increase) in $m_{h}^{*}\left(\mu_{h}\right)$ at $-0.5 \%$ [Fig. 4(a)] is related to the modification of valance bands in the vicinity of $E_{F}$ with strain as explained above. ${ }^{56}$ For the case of electron carriers, $m_{e}^{*}\left(\mu_{e}\right)$ monotonically increases (decreases) in response to biaxial strain from $+1 \%$ to $-1 \%$ correlated with the curvature alternation of LCB. As the band dispersions are not affected with the inclusion of SOC, $m^{*}, \mu$, and $\tau$ are estimated at the level of PBE. The obtained results (strain- and temperature-dependent) are summarized in Tables S1 and S2 in the supplementary material. It should be also noted that while applying the deformation potential theory, only the electron-acoustic phonon coupling, which is one of the dominant scattering mechanisms is taken into account. ${ }^{27,47,57,58}$ The other possible scattering mechanisms ${ }^{59,60}$ (i.e., polar optical phonon scattering and intervalley scattering) are not considered due to the challenges regarding the computation of electron-phonon coupling in 2D systems. ${ }^{61,62}$ For these reasons, our discussion is restricted with the intrinsic scattering based on the deformation potential theory, which yields an upper limit for the mobility. Thus, our reported transport coefficients are within the lower limit, which should increase when the different scattering mechanisms are taken into account. The variation of temperature-dependent mobility of $\mathrm{h}$-InN with the inclusion of acoustic and polar phonon scattering is shown in Fig. S4 in the supplementary material.

\section{B. Electronic transport properties}

On the basis of electronic band structure, strain-dependent electronic transport coefficients $(S, \sigma, \mathrm{PF})$ can be evaluated by solving BTE and the results are given in Fig. 5 for both charge carriers (p- and n-type) as a function of carrier concentration $(\rho)$ at $300 \mathrm{~K}$. First, in the absence of strain, the absolute value of Seebeck coefficient $\left(\left|S_{h}\right|\right.$ and $\left.\left|S_{e}\right|\right)$ decreases for both p- and n-type h-InN with $\rho$ as shown in Fig. 5(a), which is in alignment with the Mahan-Sofo theory. ${ }^{63}$ Additionally, $\left|S_{h}\right|$ is larger than $\left|S_{e}\right|$ within the studied range. For instance, the maximum value of $\left|S_{h}\right|$ is $\sim 700 \mu \mathrm{V} / \mathrm{K}$ at room temperature, which is double of $\left|S_{e}\right|$ $(\sim 350 \mu \mathrm{V} / \mathrm{K})$. This difference in $|S|$ for electron and hole carriers originates from the density of states effective mass, which is large for flatbands with high DOS around $E_{F}$. A less dispersive and degenerate HVB (hole doping) when compared with LCB (electron doping) leads to larger $|S|$. The applied biaxial strain has no significant effect on the Seebeck coefficient, therefore a similar trend is obtained with/without strain.

In contrast to $|S|$, the electronic conductivity $(\sigma)$ increases with the increasing $\rho$ and for a given $\rho, \sigma_{h}$ is smaller than $\sigma_{e}$ [Fig. 5(b)]. When strain is applied, $\sigma_{e}$ decreases (increases) monotonically with compressive (tensile) strain, following the similar trend with $\mu_{e}$ in the range of $\pm 1 \%$. In the case of p-type doping, while the effect of tensile strain on $\sigma_{h}$ is minor, it dramatically increases with compressive strain. This increase is also correlated with band convergence as it leads to multiple pathways of conducting channels within similar energy and thus enhances $\sigma_{h}$. These results suggest that p-type h-InN can demonstrate better performance than n-type system in the presence of strain. 
a)

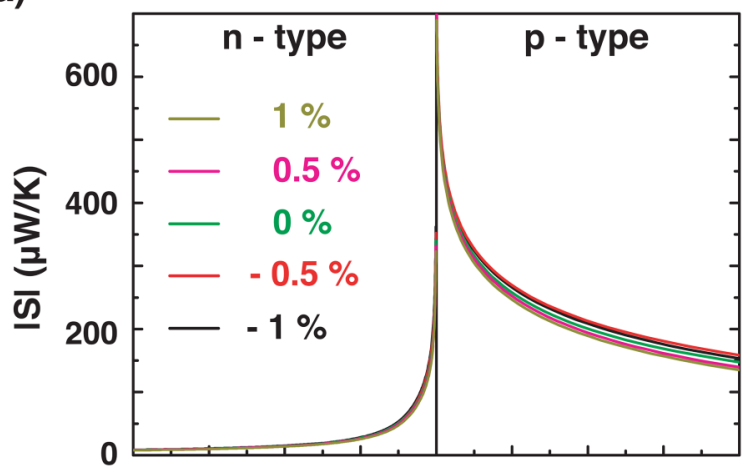

b)

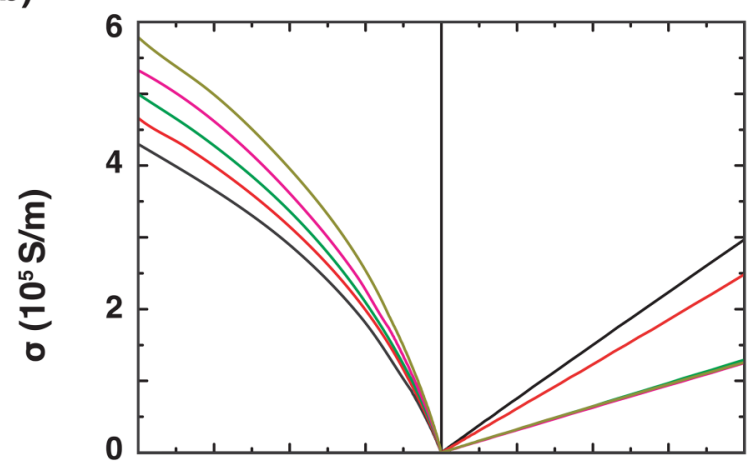

c)

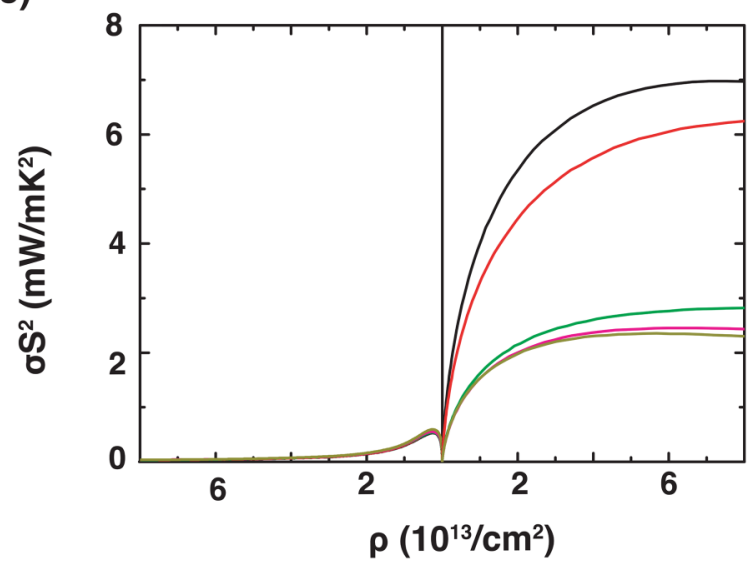

FIG. 5. (a) Seebeck coefficient $(|S|)$, (b) electrical conductivity $(\sigma)$, and (c) power factor $\left(P F=\sigma S^{2}\right)$ as a function of carrier concentration $(\rho)$ at $300 \mathrm{~K}$ for both $n$ - and $p$-type doping.

As $|S|$ decreases and $\sigma$ increases with $\rho$, their collective effect on thermoelectric response can be revealed by calculating the power factor $\left(\mathrm{PF}=\sigma S^{2}\right)$. The variation of $\mathrm{PF}$ with $\rho$ under strain is shown in Fig. 5 (c). $\mathrm{PF}_{h}$ is a significantly larger $\mathrm{PF}_{e}$, resulting in a nearly threefold magnification at the same carrier concentration in the absence of strain. While biaxial strain has minimal effect on
$\mathrm{PF}_{e}, \mathrm{PF}_{h}$ is notably enhanced under compressive strain. For instance, the maximum of $\mathrm{PF}_{h}$ obtained at $8 \times 10^{13} \mathrm{~cm}^{-2}$ hole concentration is $6.9 \mathrm{~mW} /\left(\mathrm{m} \mathrm{K}^{2}\right)$ under $-1 \%$ strain and this value is two times higher than the corresponding $\mathrm{PF}_{h}$ without strain. Therefore, the band convergence resulting from small compressive strain leads to enlarged PF.

\section{Thermal transport properties}

A low thermal conductivity is an essential factor to achieve high thermoelectric performance. The electronic contribution to the thermal conductivity $\left(\kappa_{e}\right)$ can be estimated by applying the Wiedemann-Franz law,

$$
\kappa_{e}=L \sigma T,
$$

where $L$ is the Lorentz number $\left(2.4 \times 10^{-8} \mathrm{~W} \Omega \mathrm{K}^{-2}\right.$ for free electrons), and $\sigma$ is the electrical conductivity obtained from BTE. Accordingly, $\kappa_{e}$ follows the same trend as $\sigma$, within the range of $0.31-0.76 \mathrm{~W} \mathrm{~m}^{-1} \mathrm{~K}^{-2}$ as strain applied from $+1 \%$ to $-1 \%$ at $300 \mathrm{~K}$. Its overall magnitude is much smaller than lattice thermal conductivity $\left(\kappa_{l}\right)$ and, therefore, does not affect ZT significantly.

To obtain $\kappa_{l}$, an accurate phonon spectrum should be obtained in the first place. The phonon dispersion together with phonon partial density of states (pPDOS) is shown in Fig. 6(a)
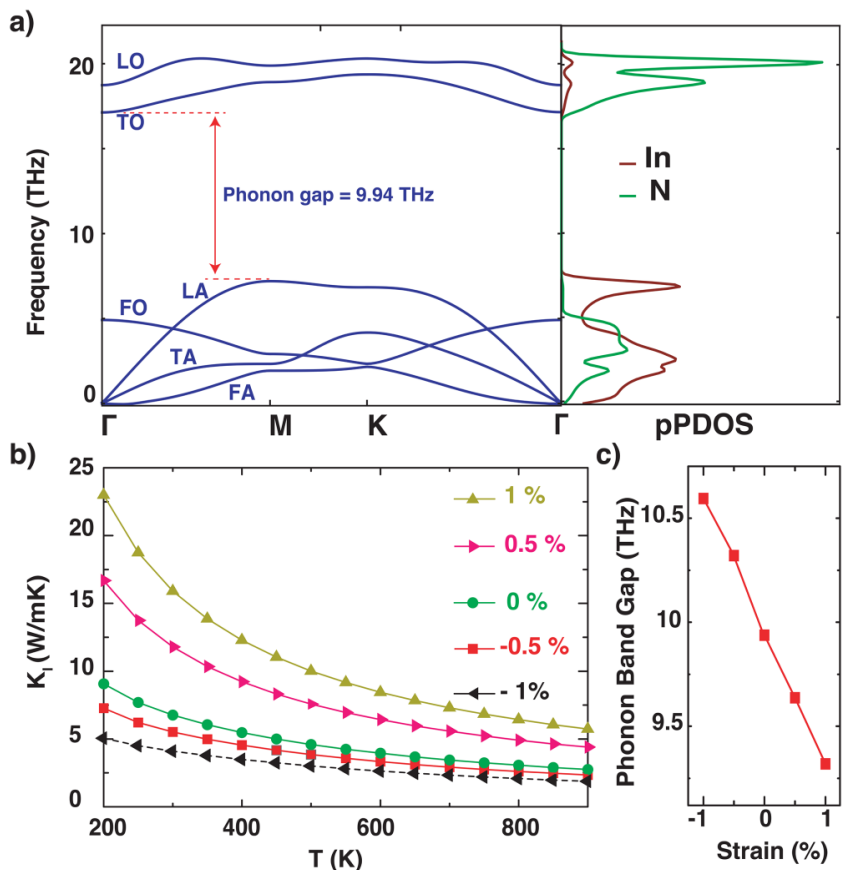

FIG. 6. (a) Phonon band structure and partial phonon density of states (pPDOS) for unstrained $\mathrm{h}-\mathrm{InN}$. (b) The variation of lattice thermal conductivity $\left(\kappa_{l}\right)$ with temperature and $(c)$ the variation of phonon bandgap with strain. $\kappa_{l}$ at $-1 \%$ is shown with dashed lines indicating the onset of imaginary frequencies at this compressive strain level. 
(the strain-dependent phonon spectrum is shown in Fig. S2 in the supplementary material). h-InN has three acoustic and three optical branches associated with two basis atoms in the primitive cell. While the transverse (TA) and longitudinal acoustic (LA) branches have a linear dispersion with the wave vector near the $\Gamma$ point, the dispersion of flexural acoustic (ZA) branch is quadratic. The flexural optical branch $(\mathrm{ZO})$ is coupled to the LA branch, which is ascribed to the planarity of group III-N monolayers. ${ }^{64,65}$ The pPDOS analyses indicate that the optical phonon branches are governed by the vibration of light $\mathrm{N}$ atom, whereas the vibration of more massive In atom dominates the high frequency of LA branch.

In the monoatomic chain model, bonding stiffness (i.e., interatomic force constant) and atomic mass in the primitive cell determine the scale of phonon dispersion frequencies. When the interatomic force constants are weaker, and the mass of the atoms is heavier, the phonon modes shift to lower frequencies. In this regard, the highest frequency of the normal mode vibration at the $\Gamma$-point for h-InN is $18 \mathrm{THz}$, which is lower than that of h-GaN $(21 \mathrm{THz})$ and h-BN $(40 \mathrm{THz}){ }^{64}$ Ascending the frequency results in a low Debye temperature ${ }^{66}\left(\Theta_{d}=\hbar \omega_{i, \max } / k_{b}\right.$ and $\omega_{i, \max }$ is the maximum phonon frequency at the zone boundary of $i$ th acoustic phonon branch), above which all the phonon modes are excited. ${ }^{67}$ Thus, a small $\Theta_{d}$ indicates an intense three-phonon scattering and leads to low $\kappa_{l}$ regarding the Slack model ${ }^{68,69}$ and clarifies the lowering of $\kappa_{l}$ from B to In in the group III-N monolayers. The $\kappa_{l}$ is calculated as $6.77 \mathrm{~W} / \mathrm{mK}$ at room temperature for h-InN and it is lower than those of h-BN $(245 \mathrm{~W} / \mathrm{mK})^{70}$ and $\mathrm{h}-\mathrm{GaN}$ $(14.9 \mathrm{~W} / \mathrm{m}),{ }^{64,71}$ in accordance with variation of $\Theta_{d}(1678,522$, and $143 \mathrm{~K}$ for h-BN, h-GaN, and h-InN, respectively). The low $\kappa_{l}$ of $\mathrm{h}$-InN can also be associated with the weak phonon harmonic interaction and strong anharmonic scattering. ${ }^{72}$ It should be noted that while calculating $\kappa_{l}$, phonon-phonon interactions are involved and electron-phonon coupling is not taken into account. Electronphonon coupling is substantial in highly doped semiconductors and metals due to possessing enough electron states around the Fermi surface. $^{73}$ Accordingly, in h-InN, this interaction is not expected to be strong. The suggested approach is applied to various 2D systems including Group III-N monolayers, ${ }^{64,74}$ and also tested on prototype materials such as graphene. ${ }^{64,74}$ The obtained results are in agreement with experimental results ${ }^{75}$ and theoretical predictions.

The variation of $\kappa_{l}$ with temperature is shown in Fig. 6(b). In the absence of strain, $\kappa_{l}$ decreases with increasing temperature following a $T^{-1}$ dependence. ${ }^{76}$ This type of variation suggests that the phonon transport scattering is mainly dominated by the Umklapp process, which modifies the thermal resistance. ${ }^{77}$ When strain is applied, $\kappa_{l}$ decreases (increases) with compressive (tensile) strain. Whereas the tensile strain leads to more isolated N-s bands, the compressive strain increases the overlapping wave functions of N-s lone-pair electrons with bonding electrons of adjacent In atoms. This induces nonlinear electrostatic forces upon thermal agitation, leading to an increase in the phonon anharmonicity and thus reducing the $\kappa_{l}{ }^{78-80}$ Therefore, $\kappa_{l}$ is reduced under the compressive strain as a result of enhanced lone-pair interaction, which is proposed as the dominant mechanism. The variation of $\kappa_{l}$ with strain is similar to other $2 \mathrm{D}$ Group III-N systems ${ }^{81}$ and this reduction is also observed in other $2 \mathrm{D}$ systems such as silicene ${ }^{82,83}$ and phosphorene. ${ }^{84}$ The phonon gap between the highest frequency of acoustic and the lowest frequency of optical branches increases (decreases) with compressive (tensile) strain [Fig. 6(c), and Fig. S3 in the supplementary material]. Compressive strain softens the phonon modes and gather them in a smaller frequency range. This increases the probability of phonon scattering and thereby reduces the phonon relaxation time. ${ }^{85,86}$ As the acoustic phonon branches primarily contribute to the thermal conductivity, softening of these modes overall results in reduced group velocity and decreased thermal conductivity. ${ }^{76}$ By contrast, tensile strain induces phonon stiffening, which often enhances the thermal conductivity. It should be noted that imaginary frequencies start to emerge at higher compressive strain levels and thus the mechanical strain should be kept low to obtain realistic results.

\section{Figure of merit}

By combining all the obtained results, the figure of merit (ZT) for doped h-InN can be estimated. ZT as a function of $\rho$ at room
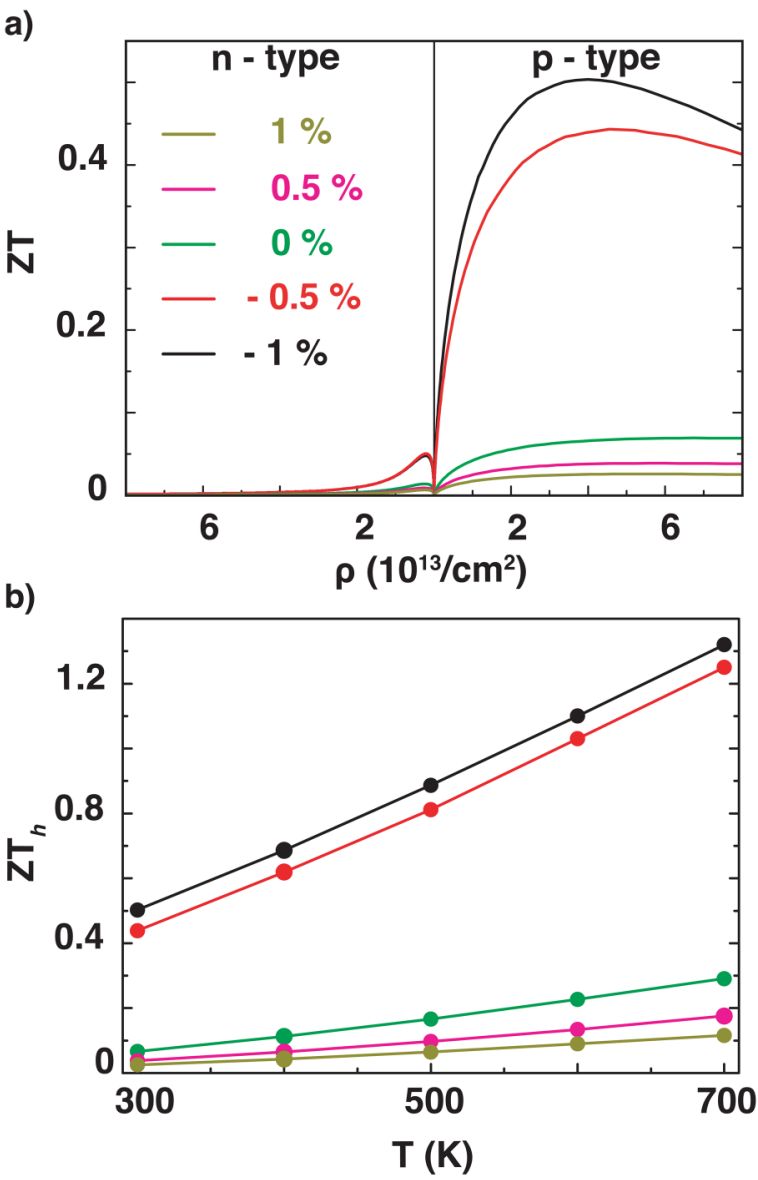

FIG. 7. (a) The variation of figure of merit (ZT) with carrier concentration for $n-$ and $p$-type doping at $300 \mathrm{~K}$ and (b) variation of strain-dependent $Z_{h}$ with temperature. 
temperature is shown in Fig. 7(a). For doping concentrations, a realistic range $\left(1 \times 10^{-13}-8 \times 10^{-13} \mathrm{~cm}^{-2}\right)$, which is attainable in $2 \mathrm{D}$ materials, is considered. ${ }^{87}$ The $\mathrm{ZT}$ of the $\mathrm{n}$ - and p-type doping is labeled as $\mathrm{ZT}_{e}$ and $\mathrm{ZT}_{h}$, respectively. In the absence of strain, the maximum of $\mathrm{ZT}_{h}$ (0.06) is threefold higher than $\mathrm{ZT}_{e}$ (0.02), which is correlated with the variation of PF [Fig. 5(c)]. While ZT increases with compressive strain for both charge carriers, it is more prominent for $\mathrm{ZT}_{h}$ where a rise by a factor of five is noticed at a strain level of $-1 \%$. The increase in $\mathrm{ZT}_{h}$ under the compressive strain is aligned with the simultaneous decrease of $\kappa_{l}$ and the increase of $\mathrm{PF}$. Such a dramatic enhancement is not obtained for $\mathrm{ZT}_{e}$ as shown in Fig. 7(a). To reveal the thermoelectric performance of h-InN at elevated temperatures, we investigate the variation of $\mathrm{ZT}_{h}$ with temperature for each strain level and the results are given in Fig. 7(b). The temperature range is selected according to the molecular dynamics results (Fig. S3 in the supplementary material), which indicates that the h-InN is dynamically stable up to $700 \mathrm{~K}$. As expected, $\mathrm{ZT}_{h}$ increases with temperature, indicating that $\mathrm{h}-\mathrm{InN}$ exhibits better performance at high temperatures. $\mathrm{ZT}_{h}$ reaches its maximum value $(0.50$ at $300 \mathrm{~K}$ and approaches up to 1.32 at $700 \mathrm{~K})$ at $-1 \%$ strain level, where band convergence is obtained. ${ }^{88}$ The obtained ZT values are larger than the conventional thermoelectric materials, for instance, $\mathrm{Bi}_{2} \mathrm{Te}_{3}(1.0),{ }^{89} \mathrm{SnSe}(0.70),{ }^{90}$ and $\operatorname{PbTe}(0.30)^{91}$

\section{CONCLUSION}

In summary, we have examined the strain-dependent thermoelectric properties of monolayer h-InN by using $a b$ initio methods together with solving Boltzmann transport equations. Our results show that the p-type system exhibits better performance than n-type, which is linked to the valence band profile near the Fermi level. Low compressive strain at the level of $-1 \%$ induces band convergence, which leads to a significant increase in PF together with a substantial decrease in $\kappa_{l}$. This modification results in high ZT, which is calculated as 0.50 at room temperature for the p-type system. Additionally, ZT enhances with elevated temperatures and reaches up to 1.32 at $700 \mathrm{~K}$, at which h-InN maintains its dynamical stability. Our results indicate that the thermoelectric performance of p-type h-InN can be significantly enhanced with applicable (low) compressive strain at realistic doping levels and suggest this system as a promising material for high-temperature thermoelectric applications.

\section{SUPPLEMENTARY MATERIAL}

See the supplementary material for additional details on electronic band structures calculated with spin-orbit coupling, straindependent phonon spectra, molecular dynamics simulations results, strain- and temperature-dependent effective mass and relaxation time values, the effect of polar phonon scattering on mobility, variation of electronic band structures under uniaxial strain and convergence tests regarding electronic, and phononic transport.

\section{ACKNOWLEDGMENTS}

The calculations were performed at TUBITAK ULAKBIM, High Performance and Grid Computing Center (TR-Grid
e-Infrastructure) and the National Center for High Performance Computing of Turkey (UHeM) under Grant No. 5007092019. This work was supported by the Scientific and Technological Research Council of Turkey (TUBITAK) under Project No. 117F241.

\section{DATA AVAILABILITY}

The data that support the findings of this study are available from the corresponding author upon reasonable request.

\section{REFERENCES}

${ }^{1}$ L. Yang, Z.-G. Chen, M. S. Dargusch, and J. Zou, Adv. Energy Mater. 8, 1701797 (2018).

${ }^{2}$ L. E. Bell, Science 321, 1457 (2008).

${ }^{3}$ J. He and T. M. Tritt, Science 357, eaak9997 (2017).

${ }^{4}$ M. S. Dresselhaus, G. Chen, M. Y. Tang, R. Yang, H. Lee, D. Wang, Z. Ren, J.-P. Fleurial, and P. Gogna, Adv. Mater. 19, 1043 (2007).

${ }^{5}$ M. G. Kanatzidis, Chem. Mater. 22, 648 (2009).

${ }^{6}$ G. Tan, F. Shi, S. Hao, H. Chi, T. P. Bailey, L.-D. Zhao, C. Uher, C. Wolverton, V. P. Dravid, and M. G. Kanatzidis, J. Am. Chem. Soc. 137, 11507 (2015).

${ }^{7}$ Y. Pei, H. Wang, and G. J. Snyder, Adv. Mater. 24, 6125 (2012).

${ }^{8}$ G. Tan, L.-D. Zhao, and M. G. Kanatzidis, Chem. Rev. 116, 12123 (2016).

${ }^{9}$ Z. Li, C. Xiao, H. Zhu, and Y. Xie, J. Am. Chem. Soc. 138, 14810 (2016).

${ }^{10}$ J. Simonson, D. Wu, W. Xie, T. Tritt, and S. Poon, Phys. Rev. B 83, 235211 (2011).

${ }^{11}$ R. Freer and A. V. Powell, J. Mater. Chem. C 8, 441 (2020).

${ }^{12}$ J. P. Heremans, M. S. Dresselhaus, L. E. Bell, and D. T. Morelli, Nat. Nanotechnol. 8, 471 (2013).

${ }^{13}$ N. T. Hung, E. H. Hasdeo, A. R. Nugraha, M. S. Dresselhaus, and R. Saito, Phys. Rev. Lett. 117, 036602 (2016).

${ }^{14}$ M.-J. Lee, J.-H. Ahn, J. H. Sung, H. Heo, S. G. Jeon, W. Lee, J. Y. Song, K.-H. Hong, B. Choi, S.-H. Lee, and M.-H. Jo, Nat. Commun. 7, 12011 (2016).

${ }^{15}$ L.-D. Zhao, S.-H. Lo, Y. Zhang, H. Sun, G. Tan, C. Uher, C. Wolverton, V. P. Dravid, and M. G. Kanatzidis, Nature 508, 373 (2014).

${ }^{16}$ C. Chang, M. Wu, D. He, Y. Pei, C.-F. Wu, X. Wu, H. Yu, F. Zhu, K. Wang, Y. Chen et al., Science 360, 778 (2018).

${ }^{17}$ K. Kanahashi, J. Pu, and T. Takenobu, Adv. Energy Mater. 10, 1902842 (2020).

${ }^{18}$ G. Qiu, S. Huang, M. Segovia, P. K. Venuthurumilli, Y. Wang, W. Wu, X. Xu, and P. D. Ye, Nano Lett. 19, 1955 (2019).

${ }^{19}$ J. Zeng, X. He, S.-J. Liang, E. Liu, Y. Sun, C. Pan, Y. Wang, T. Cao, X. Liu, C. Wang et al., Nano Lett. 18, 7538 (2018).

${ }^{20}$ D. K. Sang, T. Ding, M. N. Wu, Y. Li, J. Li, F. Liu, Z. Guo, H. Zhang, and H. Xie, Nanoscale 11, 18116 (2019).

${ }^{21}$ H. Moon, J. Bang, S. Hong, G. Kim, J. W. Roh, J. Kim, and W. Lee, ACS Nano 13 (11), 13317-13324 (2019).

${ }^{22}$ M. Buscema, M. Barkelid, V. Zwiller, H. S. van der Zant, G. A. Steele, and A. Castellanos-Gomez, Nano Lett. 13, 358 (2013).

${ }^{23}$ S. Sharma, S. Kumar, and U. Schwingenschlögl, Phys. Rev. Appl. 8, 044013 (2017).

${ }^{24}$ S. Sarikurt, D. Çakır, M. Keçeli, and C. Sevik, Nanoscale 10, 8859 (2018).

${ }^{25}$ D. Kecik, A. Onen, M. Konuk, E. Gürbüz, F. Ersan, S. Cahangirov, E. Aktürk, E. Durgun, and S. Ciraci, Appl. Phys. Rev. 5, 011105 (2018).

${ }^{26}$ V. Kumar and D. R. Roy, J. Mater. Sci. 53, 8302 (2018).

${ }^{27}$ D. Wines, F. Ersan, and C. Ataca, ACS Appl. Mater. Interfaces 12, 46416 (2020).

${ }^{28}$ I. Gorczyca, S. Łepkowski, T. Suski, N. E. Christensen, and A. Svane, Phys. Rev. B 80, 075202 (2009).

${ }^{29}$ H. Şahin, S. Cahangirov, M. Topsakal, E. Bekaroglu, E. Akturk, R. T. Senger, and S. Ciraci, Phys. Rev. B 80, 155453 (2009). 
${ }^{30}$ A. Yoshikawa, S. Che, N. Hashimoto, H. Saito, Y. Ishitani, and X. Wang, J. Vac. Sci. Technol. 26, 1551 (2008).

${ }^{31}$ J. Oila, A. Kemppinen, A. Laakso, K. Saarinen, W. Egger, L. Liszkay, P. Sperr, H. Lu, and W. Schaff, Appl. Phys. Lett. 84, 1486 (2004).

${ }^{32}$ A. Levander, T. Tong, K. Yu, J. Suh, D. Fu, R. Zhang, H. Lu, W. Schaff, O. Dubon, W. Walukiewicz et al., Appl. Phys. Lett. 98, 012108 (2011).

${ }^{33}$ R. Izaki, M. Hoshino, T. Yaginuma, N. Kaiwa, S. Yamaguchi, and A. Yamamoto, Microelectron. J. 38, 667 (2007).

${ }^{34} \mathrm{G}$. Kresse and J. Hafner, Phys. Rev. B 47, 558 (1993).

${ }^{35} \mathrm{G}$. Kresse and J. Hafner, Phys. Rev. B 49, 14251 (1994).

${ }^{36}$ G. Kresse and J. Furthmüller, Comput. Mater. Sci. 6, 15 (1996).

${ }^{37} \mathrm{G}$. Kresse and J. Furthmüller, Phys. Rev. B 54, 11169 (1996).

${ }^{38}$ J. P. Perdew, K. Burke, and M. Ernzerhof, Phys. Rev. Lett. 77, 3865 (1996).

${ }^{39}$ S. Steiner, S. Khmelevskyi, M. Marsmann, and G. Kresse, Phys. Rev. B 93, 224425 (2016).

${ }^{40}$ P. E. Blöchl, Phys. Rev. B 50, 17953 (1994).

${ }^{41}$ G. Kresse and D. Joubert, Phys. Rev. B 59, 1758 (1999).

${ }^{42} \mathrm{~K}$. Momma and F. Izumi, J. Appl. Crystallogr. 44, 1272 (2011).

${ }^{43}$ G. K. Madsen, J. Carrete, and M. J. Verstraete, Comput. Phys. Commun. 231, 140 (2018).

${ }^{44}$ G. K. Madsen and D. J. Singh, Comput. Phys. Commun. 175, 67 (2006).

${ }^{45} \mathrm{Y}$. Wu, K. Xu, C. Ma, Y. Chen, Z. Lu, H. Zhang, Z. Fang, and R. Zhang, Nano Energy 63, 103870 (2019).

${ }^{46}$ J. Bardeen and W. Shockley, Phys. Rev. 80, 72 (1950).

${ }^{47} \mathrm{H}$. J. Goldsmid et al., Introduction to Thermoelectricity (Springer, 2010), Vol. 121.

${ }^{48}$ M. Xie, S. Zhang, B. Cai, Z. Zhu, Y. Zou, and H. Zeng, Nanoscale 8, 13407 (2016).

${ }^{49}$ R. Gaska, M. Shur, A. Bykhovski, A. Orlov, and G. Snider, Appl. Phys. Lett. 74, 287 (1999).

${ }^{50}$ A. M. Ganose, A. J. Jackson, and D. O. Scanlon, J. Open Source Softw. 3, 717 (2018).

${ }^{51}$ A. Togo, L. Chaput, and I. Tanaka, Phys. Rev. B 91, 094306 (2015).

${ }^{52}$ W. Li, J. Carrete, N. A. Katcho, and N. Mingo, Comput. Phys. Commun. 185, 1747 (2014)

${ }^{53} \mathrm{~A}$. Togo and I. Tanaka, Scr. Mater. 108, 1 (2015).

${ }^{54}$ S. Yang, Y. Chen, and C. Jiang, InfoMat 3, 397 (2021).

${ }^{55}$ A. Onen, D. Kecik, E. Durgun, and S. Ciraci, Phys. Rev. B 93, 085431 (2016).

${ }^{56} \mathrm{H}$. Lv, W. Lu, D. Shao, and Y. Sun, Phys. Rev. B 90, 085433 (2014).

${ }^{57}$ H. Huang, X. Fan, D. J. Singh, and W. Zheng, J. Mater. Chem. C 8, 9763 (2020).

${ }^{\mathbf{5 8}}$ L. W. Sprague, Jr., C. Huang, J.-P. Song, and B. M. Rubenstein, J. Phys. Chem. C 123, 25437 (2019).

${ }^{59}$ V. A. Jhalani, J.-J. Zhou, and M. Bernardi, Nano Lett. 17, 5012 (2017).
${ }^{60}$ J. Wiley, Phys. Rev. B 4, 2485 (1971).

${ }^{61}$ J.-J. Zhou and M. Bernardi, Phys. Rev. B 94, 201201 (2016).

${ }^{62}$ S. H. Mir, V. K. Yadav, and J. K. Singh, ACS Omega 5, 14203 (2020).

${ }^{63}$ G. J. Snyder and E. S. Toberer, Nat. Mater. 7, 105 (2008).

${ }^{64}$ Z. Qin, G. Qin, X. Zuo, Z. Xiong, and M. Hu, Nanoscale 9, 4295 (2017).

${ }^{65}$ P. Anees, M. Valsakumar, and B. Panigrahi, Phys. Chem. Chem. Phys. 18, 2672 (2016).

${ }^{66}$ D. Morelli and J. Heremans, Appl. Phys. Lett. 81, 5126 (2002).

${ }^{67}$ T. Nakashima and Y. Umakoshi, Philos. Mag. Lett. 66, 317 (1992).

${ }^{68}$ G. A. Slack, in Solid State Physics (Elsevier, 1979), Vol. 34, pp. 1-71.

${ }^{69}$ B. Peng, H. Zhang, H. Shao, K. Xu, G. Ni, J. Li, H. Zhu, and C. M. Soukoulis, J. Mater. Chem. A 6, 2018 (2018).

${ }^{70}$ S. Illera, M. Pruneda, L. Colombo, and P. Ordejón, Phys. Rev. Mat. 1, 044006 (2017).

${ }^{71}$ G. Qin, Z. Qin, H. Wang, and M. Hu, Phys. Rev. B 95, 195416 (2017).

${ }^{72} \mathrm{H}$. Bao, J. Chen, X. Gu, and B. Cao, ES Energy Environ. 1, 16 (2018).

${ }^{73}$ Y. Huang, J. Zhou, G. Wang, and Z. Sun, J. Am. Chem. Soc. 141, 8503 (2019).

${ }^{74}$ H. Fan, H. Wu, L. Lindsay, and Y. Hu, Phys. Rev. B 100, 085420 (2019).

${ }^{75}$ O. Çakıroğlu, N. Mehmood, M. M. Çiçek, A. Aikebaier, H. R. Rasouli, E. Durgun, and T. S. Kasırga, 2D Mater. 7, 035003 (2020).

${ }^{76}$ D. Qin, X.-J. Ge, G.-Q. Ding, G.-Y. Gao, and J.-T. Lü, RSC Adv. 7, 47243 (2017).

77Z. Gao and J.-S. Wang, ACS Appl. Mater. Interfaces 12, 14298 (2020).

${ }^{78}$ M. D. Nielsen, V. Ozolins, and J. P. Heremans, Energy Environ. Sci. 6, 570 (2013).

${ }^{79}$ M. K. Jana, K. Pal, U. V. Waghmare, and K. Biswas, Angew. Chem. 128, 7923 (2016).

${ }^{80}$ J. P. Heremans, Nat. Phys. 11, 990 (2015).

${ }^{81}$ G. Qin, Z. Qin, H. Wang, and M. Hu, Nano Energy 50, 425 (2018).

${ }^{82}$ M. Hu, X. Zhang, and D. Poulikakos, Phys. Rev. B 87, 195417 (2013).

${ }^{83}$ H. Xie, T. Ouyang, E. Germaneau, G. Qin, M. Hu, and H. Bao, Phys. Rev. B 93, 075404 (2016).

${ }^{84}$ Z.-Y. Ong, Y. Cai, G. Zhang, and Y.-W. Zhang, J. Phys. Chem. C 118, 25272 (2014).

${ }^{85}$ X. Tan, G. Liu, H. Shao, J. Xu, B. Yu, H. Jiang, and J. Jiang, Appl. Phys. Lett. 110, 143903 (2017).

${ }^{86} \mathrm{H}$. Lv, W. Lu, D. Shao, H. Lu, and Y. Sun, J. Mater. Chem. C 4, 4538 (2016).

${ }^{87}$ D. K. Efetov and P. Kim, Phys. Rev. Lett. 105, 256805 (2010).

${ }^{88}$ Y. Pei, X. Shi, A. LaLonde, H. Wang, L. Chen, and G. J. Snyder, Nature 473, 66 (2011).

${ }^{89}$ L. Hicks and M. S. Dresselhaus, Phys. Rev. B 47, 12727 (1993).

${ }^{90}$ F. Q. Wang, S. Zhang, J. Yu, and Q. Wang, Nanoscale 7, 15962 (2015).

${ }^{91}$ Q. Zhang, S. Yang, Q. Zhang, S. Chen, W. Liu, H. Wang, Z. Tian, D. Broido,

G. Chen, and Z. Ren, Nanotechnology 24, 345705 (2013). 